\title{
Brain PET Imaging in Obesity and Food Addiction: Current Evidence and Hypothesis
}

\author{
Patricia Iozzo ${ }^{a} \quad$ Letizia Guiducci $^{a} \quad$ Maria Angela Guzzardi ${ }^{a}$ \\ Uberto Pagotto ${ }^{b}$ \\ ${ }^{a}$ Institute of Clinical Physiology, National Research Council (CNR), Pisa, ${ }^{b}$ Endocrinology \\ Unit, Department of Clinical Medicine, S. Orsola-Malpighi Hospital and C.R.B.A, Alma Mater \\ University of Bologna, Bologna, Italy
}

\author{
Key Words \\ Food addiction - Drug addiction - Cerebral · PET - 18F-fluorodeoxyglucose · 18F-FDG • \\ Obesity
}

\begin{abstract}
The ongoing epidemics of obesity is one main health concern of the present time. Overeating in some obese individuals shares similarities with the loss of control and compulsive behavior observed in drug-addicted subjects, suggesting that obesity may involve food addiction. Here, we review the contributions provided by the use of positron emission tomography to the current understanding of the cerebral control of obesity and food intake in humans. The available studies have shown that multiple areas in the brain are involved with the reward properties of food, such as prefrontal, orbitofrontal, somatosensory cortices, insula, thalamus, hypothalamus, amygdala, and others. This review summarizes the current evidence, supporting the concepts that i) regions involved in the somatosensory response to food sight, taste, and smell are activated by palatable foods and may be hyperresponsive in obese individuals, ii) areas controlling executive drive seem to overreact to the anticipation of pleasure during cue exposure, and iii) those involved in cognitive control and inhibitory behavior may be resistant to the perception of reward after food exposure in obese subjects. All of these features may stimulate, for different reasons, ingestion of highly palatable and energy-rich foods. Though these same regions are similarly involved in drug abusers and game-addicted individuals, any direct resemblance may be an oversimplification, especially as the heterogeneities between studies and the prevalent exclusion of sensitive groups still limit a coherent interpretation of the findings. Further work is required to comprehensively tackle the multifaceted phenotype of obesity and identify the role of food dependency in its pathophysiology.




\section{Focus of the Review}

The ongoing epidemics of obesity is one of the main health concerns of the present time. Overeating in obese individuals shares similarities with the loss of control and compulsive drug taking behavior observed in drug-addicted subjects, and the hypothesis that obesity implicates food addiction is receiving increasing attention [1]. Studies using functional or metabolic brain imaging suggest the existence of innate alterations in neural circuits controlling palatability and reward seeking in subjects with obesity. These studies have shown that multiple areas in the brain, including the orbitofrontal cortex, somatosensory cortex, temporal cortex, insula, thalamus, hypothalamus, and amygdala, are involved in the reward properties of food.

This manuscript reviews the contributions provided by the use of positron emission tomography (PET) to the current understanding of the cerebral control of obesity and food intake in humans. Special attention is paid to direct or surrogate measurements of brain glucose metabolism, because this process provides $\sim 95 \%$ of the energy required for the brain to function properly [2]. The relationship linking brain metabolism and dopaminergic activity will be analyzed in the context of obesity, since the dopamine system represents a central pathway of the neural reward circuit. Furthermore, the resemblances so far described between obesity and drug addiction will be examined.

\section{Positron Emission Tomography}

PET with the glucose analogue 2-fluoro-2-deoxyglucose $\left({ }^{18} \mathrm{~F}-\mathrm{FDG}\right)$ is the traditional technique for the direct quantification of regional brain glucose metabolism in humans, and it has been extensively used to characterize metabolic brain responses in physiological and pathological situations. PET is a nuclear imaging technique employing short-lived positronemitting radioisotopes to label molecules of interest (e.g. substrate, perfusion indicator) and visualize their fates in individual organs. Emitted positrons annihilate when combined with an electron in the body tissue, generating two $511 \mathrm{KeV}$ photons in quasi-opposite directions. Detectors are arranged all around the area of interest (e.g. the head), and events appearing nearly simultaneously in the opposite detectors are recorded and used to generate the image. After extracting from the image information pertaining to the time-activity concentration of tracer in the brain regions of interest and in blood - the latter reflecting the amount of tracer available for tissue extraction - mathematical modeling is used to quantify biochemical processes within the target tissue area. Brain maps can be constructed to represent the distribution of the parameter under study, e.g. glucose uptake rate, perfusion or receptor binding, and availability.

${ }^{18} \mathrm{~F}-\mathrm{FDG}$ is the most commonly adopted PET tracer. It is characterized by the substitution of a fluorine-18 (positron-emitting) radionuclide in place of the hydroxyl group bound to the second carbon of the glucose carbon chain. As compared to glucose, this tracer is transported into cells and phosphorylated, but cannot undergo glycolysis and therefore accumulates in the brain in proportion with cerebral glucose consumption, providing images of elevated visual quality. ${ }^{18} \mathrm{~F}-\mathrm{FDG}$-PET imaging is functional, quantitative, and specific to glucose metabolism. Blood perfusion measurements (PET with the perfusion tracer $\mathrm{H}_{2}{ }^{15} \mathrm{O}$ or single photon emission-computed tomography, i.e. SPECT) and blood oxygenation leveldependent magnetic resonance imaging (BOLD MRI) [3] have been used as indirect indicators of brain metabolism. In addition, a variety of labeled PET ligands have been developed to target receptor binding of neurotransmitters involved in the regulation of brain metabolism, reward, and behavioral control [4]. 
PET imaging can be conducted under different experimental conditions, depending on the target process. In the field of obesity research, PET studies have been conducted during fasting, meal ingestion, and palatable food (gustatory, olfactory, visual) stimulation. Account has to be taken of the fact that palatability and hunger/satiety are distinct modulators of food reward, since the former is solely dependent on the taste, smell, and sight of food, whereas the latter incorporates the additional effects of gastrointestinal distension and signaling plus hormone-substrate changes occurring after meal ingestion. Cerebral responses to insulin and glucagon-like-peptide 1 (GLP-1) have been addressed by combining ${ }^{18}$ F-FDG-PET imaging with the insulin clamp technique [5-7].

\section{Brain Glucose Metabolism and Blood Flow in Obesity: The Cerebral Response to Palatable Food}

In 2002, Wang et al. [8] conducted a cross-sectional study to evaluate brain metabolism in 10 morbidly obese (mean BMI $51 \mathrm{~kg} / \mathrm{m}^{2}$ ) and 14 lean subjects $\left(21 \mathrm{~kg} / \mathrm{m}^{2}\right)$. The observed tendency of global brain glucose metabolism to be greater in obese than lean subjects fell just short of conventional statistical significance $(p=0.051)$. Subregional analysis did demonstrate significant differences in the parietal cortex (postcentral gyrus, Brodmann's area), upper cerebellum, and precuneus. The former area in the parietal cortex is involved with direct taste perception, as it contains the somatosensory maps of the mouth, lips, and tongue. The cerebellum has been suggested to play an important role in the control of food intake, since glucose uptake there increased during bar pressing behavior anticipating food reward in rats [9], while stimulation of the cerebellar interpositus nucleus affected the neuronal activity of the lateral hypothalamic area [10]. The involvement of the above brain regions in the physiological cerebral response to palatable food sensing was confirmed in a cross-over study in 12 healthy lean subjects (mean BMI $24 \mathrm{~kg} / \mathrm{m}^{2}$ ) who were exposed to the sight, smell, and taste of either a palatable or neutral (non-food) stimuli on two separate days [11]. ${ }^{18}$ F-FDG-PET scans were performed and questionnaires for hunger rating were filled in on each occasion. Compared with the neutral intervention condition, the food presentation significantly increased whole brain metabolism $(+24 \%, \mathrm{p}<0.01)$. The largest increases occurred in the left and right postcentral gyrus (parietal somatosensory area, map of the tongue), left superior temporal, left anterior insula (primary gustatory area), and left orbitofrontal cortex (secondary gustatory cortex). Palatable food presentation was paralleled by a significant increase in the ratings of hunger and desire for food, which correlated with the metabolic changes observed in the right orbitofrontal cortex. A greater metabolism in somatosensory areas may translate in an enhanced cerebral sensitivity towards palatable foods, promoting the preferential consumption of calorie-rich foods, thus fostering the progression of obesity. Together, the above studies suggest that the somatosensory area - which is activated upon food perception/anticipation and associated with increased hunger in lean subjects - may be chronically activated in obese individuals, independent of food presentation.

A recent study identified a significant inverse correlation between BMI (range 19-37 $\mathrm{kg} / \mathrm{m}^{2}$ ) and baseline brain glucose metabolism in prefrontal regions and in the anterior cingulate gyrus [12], i.e. a lower metabolism in these cerebral areas was observed in individuals with a greater BMI. The prefrontal and, more specifically, the orbitofrontal cortex participate in the learning of associations between visual, olfactory, and taste stimuli, attributing motivational value to gustatory stimuli and expectation of food reward. Processing of the information occurs through projections from the insula, striatum, and amygdala, and these areas may be relevant in the inhibitory control of food intake. Different 
from the primary somatosensory area, which relates to the intensity and identity of the food, neurons in the orbitofrontal cortex respond to the sight of food in proportion to its reinforcing properties [13], and they are therefore concerned with the cognitive level of perceived reward and anticipated pleasure. Therefore, a reduced metabolism in this area in obese individuals may require a compensatory increase in food consumption to achieve a given sensation of reward. Considering the easy availability of food in the developed world, the capacity to refrain is especially important in preventing food overconsumption and body weight excess, and the lack of activation in these areas may translate as a weak reward sensing.

However, all studies mentioned do not take in consideration the multifaceted nature of the obesity phenotype, encompassing a variety of behavioral and psychiatric deviations. Importantly, when studying brain regions involved in cognition, motivation, and capability to refrain from food temptation the careful psychological characterization of study subjects may help distinguish common obesity-related abnormalities from those confined to a restricted phenotype. This is demonstrated by a scintigraphy study performed during the exposure to food, in which an increase in the cerebral blood flow being greater in the left than in the right hemisphere, especially in the frontal and prefrontal regions, that correlates with the increase in the feeling of hunger was found in obese binge eating women as compared with similarly obese non-binge eating women [14].

\section{Brain Metabolism and Blood Flow in Obesity: The Integrated Response to Meal Consumption}

Meal consumption, including quality and quantity of food, is regulated by integration of the palatability and hunger/satiety sensations. Palatability is independent of meal size and mostly centered on expectation, motivation, and reward, which are modulated in part by food taste, smell, and sight. Beyond these elements, meal ingestion involves gastric responses, including gastrointestinal distension due to meal size and texture, neurologic reflexes, and gastrointestinal hormones. In addition, meals result in circulating substrate changes which affect brain metabolism and peripheral hormone levels. Though this complex situation reflects the real life situation, when these aspects are studied together, it is difficult to isolate the contribution of each to the modulation of energy intake mediated by brain metabolism.

Studies investigating the effects of hunger and satiation by PET have been mostly concerned with brain perfusion. In 11 healthy individuals [15], responses to a 36-hour fasting period were compared with those following the ingestion of a liquid meal over a 25-min oral infusion period. Hunger was associated with increased blood flow in the proximity of the hypothalamic, thalamic, limbic, and paralimbic areas and the putamen, precuneous, and cerebellum, which is consistent with the previously mentioned implication of these regions in the control of food intake. As mentioned above, several of these regions are already metabolically active in the fasting, nonstimulated state in obese individuals. Satiation was associated with increased activity in the somatosensory and prefrontal cortices, which is consistent with the responses of brain glucose metabolism to palatability as mentioned previously. Part of the changes may simply relate to gastric distension since the application of pure mechanical distension of the stomach (by using air or water) also causes changes in neuronal activity in several brain regions, including the insula, anterior cingulate, caudate nucleus, thalamus, brainstem, cerebellum, superior temporal gyrus, occipital gyrus, and inferior frontal gyrus, all of which show a progressive increase in activation with increasing intensity of the distending stimulus [16-18]. 
Iozzo et al.: Brain PET Imaging in Obesity and Food Addiction: Current Evidence and Hypothesis

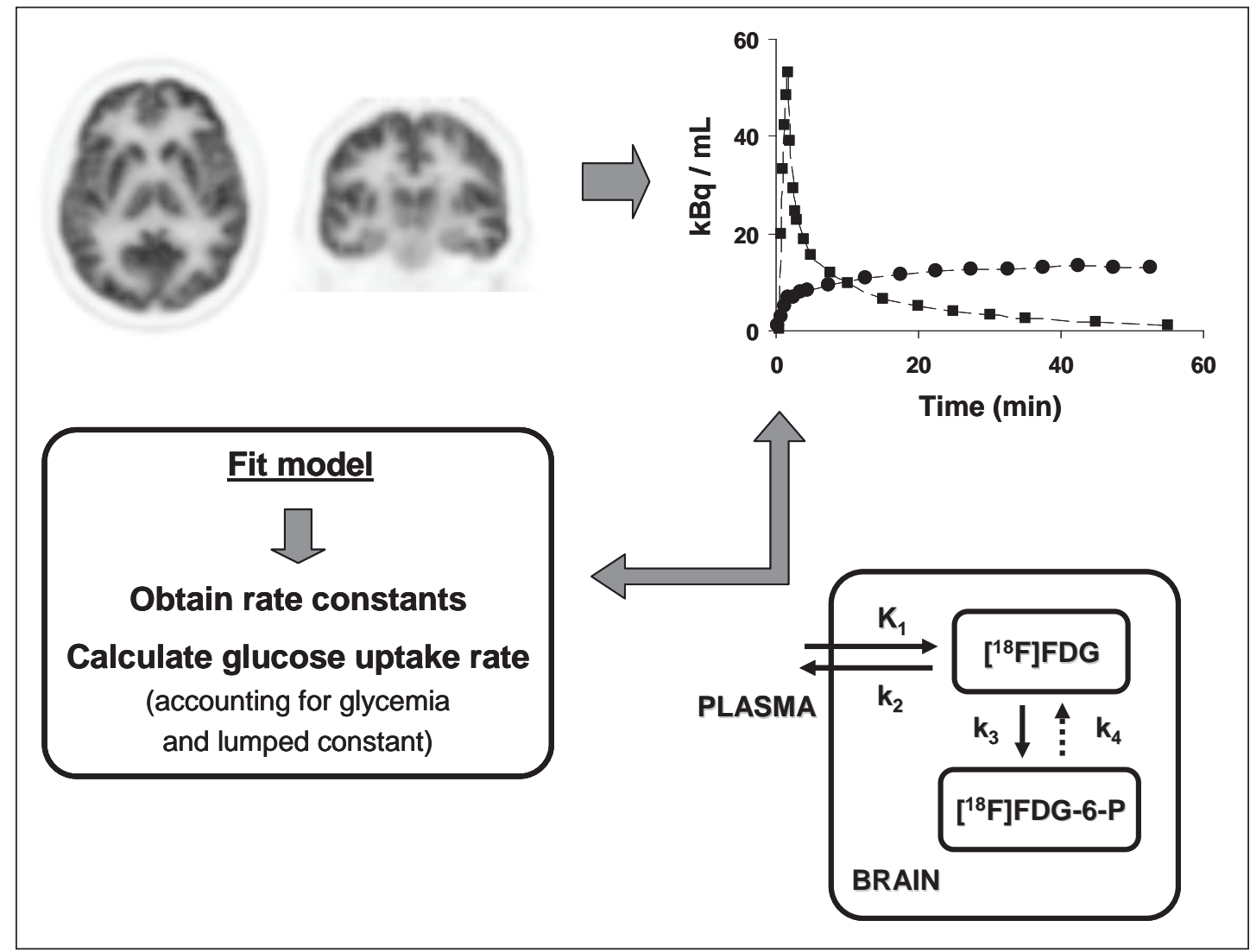

Fig. 1. A representative ${ }^{18} \mathrm{~F}-\mathrm{FDG}$-PET image is shown in a transaxial and in a coronal plane (top left), together with the steps required in the quantification analysis, i.e. the obtainment of time-activity curves describing the kinetics of ${ }^{18} \mathrm{~F}$-FDG in tissue and blood (top right), the model used to represent the movement of ${ }^{18} \mathrm{~F}$-FDG between compartments (bottom right) and to estimate the transfer rate constants, and the subsequent use of rate constants to obtain glucose uptake data in the selected brain region(s) of interest (bottom left). The lumped constant term supposedly corrects for differences in transport and phosphorylation between the tracer and glucose. The $\mathrm{k}_{4}$ term is adjacent to a dashed arrow since the breakdown and loss of ${ }^{18} \mathrm{~F}-\mathrm{FDG}-6$ phosphate $\left({ }^{18} \mathrm{~F}-\mathrm{FDG}-6-\mathrm{P}\right)$ is assumed to be negligible within the time frame of most PET studies.

In obese compared with lean men [19], the changes in the prefrontal region, limbic/ paralimbic areas, temporal and occipital cortices, and cerebellum induced by meal ingestion were more pronounced, and the changes in the hypothalamus and thalamus were blunted. Though some sex-related differences in the cerebral flow response to satiety have been described [20], the effects of obesity were similar in women [21], suggesting that the activation of the prefrontal (inhibitory) region may represent a generic compensatory response to control the more sensitive orexigenic areas observed in the obese when hungry.

It is important to recognize that meal energy contents in the above studies were based on daily energy expenditure, and therefore they were greater in obese than in lean subjects (e.g. $954 \pm 54 \mathrm{kcal}$ for the obese vs. $725 \pm 105$ for the lean men [19]). Once this factor was carefully accounted for by comparing satiating versus fixed meal sizes in obese and lean individuals [22], the greater activation in the right medial orbitofrontal cortex was proportional and could be attributed to satiation due to meal size, regardless of body weight category. Obesity per se, however, resulted in significantly less activation in the left dorsolateral prefrontal cortex in response to both satiating and fixed meals. Successful dieters 
maintaining weight loss for at least 3 months showed a greater activation in the dorsal prefrontal cortex, dorsal striatum, and anterior cerebellar lobe as well as a lower activation in the orbitofrontal cortex in response to a meal as compared to BMI-matched non-dieters [23]. These studies may imply that the hyporesponsiveness of the orbitofrontal cortex following weight loss may weaken the inhibitory control in these susceptible individuals and predispose them to eventually regain the lost weight.

There are other important components of the response to food ingestion, which differ in lean and obese individuals, and are separate from aspects of satiation or pure motivation and reward. These components include the changes in insulin, gastrointestinal hormones, adipokines, and substrate concentrations presented to the brain. For example, the concomitant rise in insulin or the decline in circulating fatty acid concentrations following liquid meal infusion both closely correlated with the observed changes in regional cerebral blood flow [15]. In addition, adipose tissue masses relate with brain responses to a meal [22], implying the possible involvement of signaling molecules, e.g. adipokines. Such linked metabolic interactions make it impossible to distinguish the separate contribution of satiety, palatability, and hormone/substrate changes in modulating brain responses to a meal.

Insulin is the primary hormone secreted after food ingestion to promote energy storage and glucose consumption in peripheral insulin-sensitive tissues. In order to characterize the effects of hyperinsulinemia in isolation, a standardized insulin infusion can be administered to mimic postprandial hyperinsulinemia, while maintaining euglycemia by appropriate glucose infusion. This infusion leads to a suppression in circulating free fatty acids, which is consistent with that observed upon eating. We have recently compared fasted and insulinmediated cerebral blood flow and glucose metabolism by PET scanning with $\mathrm{H}_{2}{ }^{15} \mathrm{O}$ and ${ }^{18} \mathrm{~F}-\mathrm{FDG}$ in obese (glucose-intolerant) and leaner subjects (with normal glucose tolerance) [6]. Consistent with previous studies [5], hyperinsulinemia did not influence cerebral metabolism in lean individuals or cerebral blood flow in either group. However, brain glucose metabolism was selectively increased for the obese, glucose-intolerant group, particularly in the right posterior insula. Though further studies are needed to distinguish whether brain responses were mostly due to obesity or chronic hyperglycemia, these data suggest that insulin action may be partly implicated in the meal-induced hypermetabolism observed in obese individuals, especially in the somatosensory cerebral regions. In addition, these data cautions against the assumption that cerebral blood flow directly reflects glucose metabolism.

GLP-1 is an incretin hormone produced by the entero-endocrine L-cells in the gut and by the brain. It stimulates insulin secretion and inhibits glucagon secretion in the presence of glucose, inhibits gastric emptying, and reduces appetite and food intake. The infusion of this hormone has been implicated in the down-regulation of brain glucose transport [7], suggesting that the hormone may limit excursions in brain glucose concentrations after a meal. However, in the above studies that compared obese and lean individuals [19], GLP-1 was not found to be modified by the liquid meal infusion.

\section{Brain Glucose Metabolism and Dopamine Receptors in Obesity}

Dopamine (DA) is one of the neurotransmitters that modulate prefrontal activity, thus regulating executive function. DA is involved in the regulation of eating behavior and a central player in the mesolimbic reward circuit, and negative emotions have been associated with a decrease in DA activity in conjunction with the loss of motivation to seek reward [24].

The type 2 dopamine receptor (D2) ligand ${ }^{11} \mathrm{C}$-raclopride has been used in PET imaging studies. The D2 receptor availability and DA response to food palatability (sight, smell of 
warmed food, and taste of a variety of foods) were evaluated in healthy individuals [25]. Tests were performed to score the degree of cognitive restraint towards food intake to control weight, emotional distress (i.e. the tendency to eat when exposed to negative emotions), and sensitivity to external appetitive food stimuli. A positive association was observed in the dorsal striatum between the restraint factor and the DA response to food stimulation. The authors suggested that a stronger restraining effort in these subjects may reflect an attempt to cognitively counteract the higher DA response, since the latter may underlie a greater appeal of food stimuli. This is in keeping with their previous report showing that the same DA response was associated with the 'desire for food' and with the perception of 'hunger' [26]. Importantly, a negative correlation was shown between the emotionality factor and the baseline D2 receptor availability in the dorsal striatum, suggesting that emotional stress may be associated with an impaired stimulation of foodmediated reward, enhancing the risk of overeating. This is consistent with the evidence that medications blocking D2 receptors increase food intake and the risk of obesity [27, 28].

To gain insight into the mechanisms by which low D2 receptor availability increases the risk of overeating, Volkow et al. [4] combined PET imaging of ${ }^{18} \mathrm{~F}$-FDG and of ${ }^{11} \mathrm{C}$-raclopride in 10 morbidly obese (BMI $51 \mathrm{~kg} / \mathrm{m}^{2}$ ) and 12 lean individuals (BMI $25 \mathrm{~kg} / \mathrm{m}^{2}$ ). They found that striatal D2 receptor availability was reduced by 13\% in obese compared with in lean subjects $(\mathrm{p}<0.05)$. This is consistent with previous evidence showing a progressive reduction in D2 receptor binding in proportion to an increasing BMI [29]. In addition, in morbidly obese subjects D2 receptor availability was positively associated with glucose uptake in prefrontal regions (orbitofrontal cortex and anterior cingulated gyrus). This is in accordance with another recent report by the same authors [12], extending the association to a more physiological BMI range of $19-37 \mathrm{~kg} / \mathrm{m}^{2}$ in 21 healthy individuals. The authors suggested that the relationship between BMI and prefrontal metabolism may reflect disrupted dopaminergic activity.

In fact, DA signaling projects from the ventral tegmental area and substantia nigra to the striatum which is implicated in the reinforcing and motivational effects of food. Projections from the striatum to the prefrontal and somatosensory cortices may affect the metabolic activity of these regions. In turn, the prefrontal cortex sends projections to the striatum, and prefrontal glucose metabolism may regulate DA striatal activity [30]. Thus, these areas and processes may operate in a reciprocal feedback system. Brain glucose metabolism is also sensitive to peripheral hormones and substrates influencing food desire and energy intake, both of which are modified by meals and obesity, and it is therefore the ideal pathway to receive and integrate local dopaminergic and peripheral signals.

\section{Do PET Studies Support the Concept That Obesity Is Caused by an 'Addiction' to Food?}

Both obesity and addiction share the inability to restrain behavior in spite of an awareness of detrimental health and social consequences. The difference between individuals in their ability to exert self-inhibition and self-control determines the outcome in both conditions. However, in contrast to drug, alcohol, and cigarette use, and also gameplaying, eating is essential for survival. Consequently, both overeating and living in a foodrich environment have no legal or moral constraints. As a matter of fact, obesity has reached epidemic proportions.

Obesity and substance addiction share common features in brain metabolism. Similar to observations in obese individuals, a significant association between D2 receptor availability and metabolism in prefrontal regions was observed in drug-addicted subjects 
(cocaine, methamphetamine, and alcohol) [31-33]. The same finding in individuals at high familial risk for alcoholism [34] suggests that these abnormalities precede the change in behavior. A defective baseline orbitofrontal metabolism could underlie compulsiveness and lack of control and promote compensatory overeating and substance abuse.

On the other hand, a greater response of the orbitofrontal cortex to food or drug exposure may be involved with further drive and desire because of the pleasure of the anticipated reward. In fact, cocaine craving increased brain metabolism in the orbitofrontal cortex upon stimulant or drug cue administration in cocaine-addicted subjects [35-37].

The involvement of the prefrontal cortex in addictive behaviors is not specific to a form of addiction and not caused by a direct effect of a given drug or food on the brain. In fact, internet game overuse, another emerging disorder characterized by lack of impulse control and poor reward processing, but not involving the intake of exogenous substances, was also associated with abnormal glucose metabolism in the orbitofrontal cortex, striatum, and sensory regions [38].

This does not imply that chronic drug consumption is non-harmful with respect to brain metabolism. On the contrary, it may severely amplify the damage and compromise previously healthy regions. A recently published study conducted in cocaine-naïve monkeys has provided important evidence in this regard, since cocaine-induced increases in brain metabolism were initially restricted to the prefrontal cortex, but they expanded throughout the frontal cortex and the striatum as cocaine exposure progressed. Cocaine-induced activation was far less robust following withdrawal, possibly suggesting that larger doses of the drug would be required to achieve a similar brain activation and reward once the addictive state is achieved [39].

\section{Conclusions and Perspectives}

One hypothesis derived from the various findings is that in obese individuals, those regions involved in the somatosensory response to food sight, taste, and smell - if hyperresponsive - increase the appeal of calorie-rich foods, while areas controlling executive drive may overreact to the anticipation of pleasure. At the same time, regions involved in cognitive control and inhibitory behavior may be resistant to the perception of reward after food exposure, thereby requiring a compensatory increase in food ingestion. However, important differences in study designs limit interpretation of the findings. Even when a single imaging modality is used, such as PET as in the present case, the approaches have ranged from indirect brain activation studies by perfusion to direct quantification of brain glucose metabolism, and one should exercise caution in giving these procedures equal status. Standardized use of ${ }^{18}$ F-FDG would have the advantage that the signal can be reconducted to a specific process (glucose uptake) vital for the brain and functionally connected with dopaminergic activity. This tracer is also widely accessible, allowing for study comparison and integration between centers. Account should be taken to combine imaging results with a thorough evaluation of psychosocial and hormonal profiles (e.g. activation of the hypothalamus-pituitary-adrenal axis and sympathetic tone, adipokines, and gastrointestinal peptides), which play roles in the pathophysiology of obesity and obesity-related complications and are also strongly and reciprocally related with brain function.

Current data on obese and drug-addicted individuals are insufficient to postulate common mechanisms for emotional food dependency and addiction. Furthermore, most studies have conducted a psychological screening to exclude patients with problematic eating attitudes or other substance abuse. It is not clear how many subjects have been excluded for these reasons, but such discrimination limit the ability to detect similarities 
between obesity and food addiction. In addition, weight loss studies have been focused on successful dieters, whereas food addiction may be more logically associated with the failure to achieve and maintain the target weight by restricting food choices (i.e., quitting the subjectively most rewarding foods) beyond the number of calories. Stratification (rather than exclusion) of individuals based on a psychological evaluation to distinguish patients with compulsive and addictive features, whose overeating responds to emotional distress, from those who are merely sensitive to external appetitive food stimuli would provide a comprehensive and realistic description of the heterogeneity of the obesity phenotype. Weight loss interventions in psychologically stratified groups may allow distinction between primary and secondary (BMI-dependent) brain abnormalities, based on the hypothesis that individuals showing addictiveness may have persisting aberrations in cerebral metabolism after weight loss and manifest symptoms upon withdrawal of energy-enriched foods, including the extreme difficulty to abstain from the preferred foods and craving.

\section{Acknowledgements}

This research was supported by a grant from the European Union (NEUROFAST FPVIIKBBE-2009-3-245009) to P.I. and U.P.

\section{Disclosure Statement}

The authors have no conflict to disclose in relation to this work.

\section{References}

1 Lutter M, Nestler EJ: Homeostatic and hedonic signals interact in the regulation of food intake. J Nutr 2009; 139:629-632.

2 Phelps ME: Positron emission tomography provides molecular imaging of biological processes. Proc Natl Acad Sci U S A 2000;97:9226-9233.

3 Goldstone AP, Prechtl de Hernandez CG, Beaver JD, Muhammed K, Croese C, Bell G, Durighel G, Hughes E, Waldman AD, Frost G, Bell JD: Fasting biases brain reward systems towards high-calorie foods. Eur J Neurosci 2009;30:1625-1635.

- 4 Volkow ND, Wang GJ, Telang F, Fowler JS, Thanos PK, Logan J, Alexoff D, Ding YS, Wong C, Ma Y, Pradhan K: Low dopamine striatal D2 receptors are associated with prefrontal metabolism in obese subjects: possible contributing factors. Neuroimage 2008;42:1537-1543.

5 Hasselbalch SG, Knudsen GM, Videbaek C, Pinborg LH, Schmidt JF, Holm S, Paulson OB: No effect of insulin on glucose blood-brain barrier transport and cerebral metabolism in humans. Diabetes 1999;48:19151921.

- 6 Hirvonen J, Virtanen KA, Nummenmaa L, Hannukainen JC, Honka MJ, Bucci M, Nesterov SV, Parkkola R, Rinne J, Iozzo P, Nuutila P: Effects of insulin on brain glucose metabolism in impaired glucose tolerance. Diabetes 2011;60:443-447.

$\checkmark 7$ Lerche S, Brock B, Rungby J, Bøtker HE, Møller N, Rodell A, Bibby BM, Holst JJ, Schmitz O, Gjedde A: Glucagonlike peptide-1 inhibits blood-brain glucose transfer in humans. Diabetes 2008;57:325-331.

$\checkmark 8$ Wang GJ, Volkow ND, Felder C, Fowler JS, Levy AV, Pappas NR, Wong CT, Zhu W, Netusil N: Enhanced resting activity of the oral somatosensory cortex in obese subjects. Neuroreport 2002;13:1151-1155.

- 9 Morimoto A, Suzumi M, Sakata Y, Murakami N: Activation of brain regions in rats during food-intake operant behavior. Physiol Behav 1984;33:965-968.

-10 Pu YM, Wang JJ, Wang T, Yu QX: Cerebellar interpositus nucleus modulates neuronal activity of lateral hypothalamic area. Neuroreport 1995;6:985-988.

11 Wang GJ, Volkow ND, Telang F, Jayne M, Ma J, Rao M, Zhu W, Wong CT, Pappas NR, Geliebter A, Fowler JS: Exposure to appetitive food stimuli markedly activates the human brain. Neuroimage 2004;21:1790-1797.

12 Volkow ND, Wang GJ, Telang F, Fowler JS, Goldstein RZ, Alia-Klein N, Logan J, Wong C, Thanos PK, Ma Y, Pradhan K: Inverse association between BMI and prefrontal metabolic activity in healthy adults. Obesity (Silver Spring) 2009;17:60-65. 
13 Rolls ET: Taste and olfactory processing in the brain and its relation to the control of eating. Crit Rev Neurobiol 1997;11:263-287.

14 Karhunen LJ, Vanninen EJ, Kuikka JT, Lappalainen RI, Tiihonen J, Uusitupa MI: Regional cerebral blood flow during exposure to food in obese binge eating women. Psychiatry Res 2000;99:29-42.

15 Tataranni PA, Gautier JF, Chen K, Uecker A, Bandy D, Salbe AD, Pratley RE, Lawson M, Reiman EM, Ravussin E: Neuroanatomical correlates of hunger and satiation in humans using positron emission tomography. Proc Natl Acad Sci U S A 1999;96:4569-4574.

16 Vandenbergh J, Dupont P, Fischler B, Bormans G, Persoons P, Janssens J, Tack J: Regional brain activation during proximal stomach distention in humans: A positron emission tomography study. Gastroenterology 2005; 128:564-573.

$\checkmark 17$ Stephan E, Pardo JV, Faris PL, Hartman BK, Kim SW, Ivanov EH, Daughters RS, Costello PA, Goodale RL: Functional neuroimaging of gastric distention. J Gastrointest Surg 2003;7:740-749.

18 Ladabaum U, Minoshima S, Hasler WL, Cross D, Chey WD, Owyang C: Gastric distention correlates with activation of multiple cortical and subcortical regions. Gastroenterology 2001;120:369-376.

19 Gautier JF, Chen K, Salbe AD, Bandy D, Pratley RE, Heiman M, Ravussin E, Reiman EM, Tataranni PA: Differential brain responses to satiation in obese and lean men. Diabetes 2000;49:838-846.

20 Del Parigi A, Chen K, Gautier JF, Salbe AD, Pratley RE, Ravussin E, Reiman EM, Tataranni PA: Sex differences in the human brain's response to hunger and satiation. Am J Clin Nutr 2002;75:1017-1022.

-21 Gautier JF, Del Parigi A, Chen K, Salbe AD, Bandy D, Pratley RE, Ravussin E, Reiman EM, Tataranni PA: Effect of satiation on brain activity in obese and lean women. Obes Res 2001;9:676-684.

22 Le DS, Pannacciulli N, Chen K, Del Parigi A, Salbe AD, Reiman EM, Krakoff J: Less activation of the left dorsolateral prefrontal cortex in response to a meal: a feature of obesity. Am J Clin Nutr 2006;84:725-731.

-23 Del Parigi A, Chen K, Salbe AD, Hill JO, Wing RR, Reiman EM, Tataranni PA: Successful dieters have increased neural activity in cortical areas involved in the control of behavior. Int J Obes (Lond) 2007;31:440-448.

-24 Schmidt K, Nolte-Zenker B, Patzer J, Bauer M, Schmidt LG, Heinz A: Psychopathological correlates of reduced dopamine receptor sensitivity in depression, schizophrenia, and opiate and alcohol dependence. Pharmacopsychiatry 2001;34:66-72.

25 Volkow ND, Wang GJ, Maynard L, Jayne M, Fowler JS, Zhu W, Logan J, Gatley SJ, Ding YS, Wong C, Pappas N: Brain dopamine is associated with eating behaviors in humans. Int J Eat Disord 2003;33:136-142.

26 Volkow ND, Wang GJ, Fowler JS, Logan J, Jayne M, Franceschi D, Wong C, Gatley SJ, Gifford AN, Ding YS, Pappas N: 'Nonhedonic' food motivation in humans involves dopamine in the dorsal striatum and methylphenidate amplifies this effect. Synapse 2002;44:175-180.

-27 Inoue K, Kiriike N, Fujisaki Y, Okuno M, Ito H, Yamagami S: D2 receptors in the ventrolateral striatum are involved in feeding behavior in rats. Pharmacol Biochem Behav 1995;50:153-161.

-28 de Leeuw van Weenen JE, Auvinen HE, Parlevliet ET, Coomans CP, Schröder-van der Elst JP, Meijer OC, Pijl $\mathrm{H}$ : Blocking dopamine D2 receptors by haloperidol curtails the beneficial impact of calorie restriction on the metabolic phenotype of high-fat diet induced obese mice. J Neuroendocrinol 2011;23:158-167.

-29 Wang GJ, Volkow ND, Logan J, Pappas NR, Wong CT, Zhu W, Netusil N, Fowler JS: Brain dopamine and obesity. Lancet 2001;357:354-357.

-30 Murase S, Grenhoff J, Chouvet G, Gonon FG, Svensson TH: Prefrontal cortex regulates burst firing and transmitter release in rat mesolimbic dopamine neurons studied in vivo. Neurosci Lett 1993;157:53-56.

-31 Volkow ND, Chang L, Wang GJ, Fowler JS, Ding YS, Sedler M, Logan J, Franceschi D, Gatley J, Hitzemann R, Gifford A, Wong C, Pappas N: Low level of brain dopamine D2 receptors in methamphetamine abusers: association with metabolism in the orbitofrontal cortex. Am J Psychiatry 2001;158:2015-2021.

-32 Volkow ND, Wang GJ, Telang F, Fowler JS, Logan J, Jayne M, Ma Y, Pradhan K, Wong C: Profound decreases in dopamine release in striatum in detoxified alcoholics: possible orbitofrontal involvement. J Neurosci 2007; 27:12700-12706.

-33 Volkow ND, Fowler JS, Wang GJ, Hitzemann R, Logan J, Schlyer DJ, Dewey SL, Wolf AP: Decreased dopamine D2 receptor availability is associated with reduced frontal metabolism in cocaine abusers. Synapse 1993; 14:169-177.

-34 Volkow ND, Wang GJ, Begleiter H, Porjesz B, Fowler JS, Telang F, Wong C, Ma Y, Logan J, Goldstein R, Alexoff D, Thanos PK: High levels of dopamine D2 receptors in unaffected members of alcoholic families: possible protective factors. Arch Gen Psychiatry 2006;63:999-1008.

-35 Bonson KR, Grant SJ, Contoreggi CS, Links JM, Metcalfe J, Weyl HL, Kurian V, Ernst M, London ED: Neural systems and cue-induced cocaine craving. Neuropsychopharmacology 2002;26:376-386.

36 Grant S, London ED, Newlin DB, Villemagne VL, Liu X, Contoreggi C, Phillips RL, Kimes AS, Margolin A: Activation of memory circuits during cue-elicited cocaine craving. Proc Natl Acad Sci U S A 1996;93:1204012045.

-37 Wang GJ, Volkow ND, Fowler JS, Cervany P, Hitzemann RJ, Pappas NR, Wong CT, Felder C: Regional brain metabolic activation during craving elicited by recall of previous drug experiences. Life Sci 1999;64:775-784.

-38 Park HS, Kim SH, Bang SA, Yoon EJ, Cho SS, Kim SE: Altered regional cerebral glucose metabolism in internet game overusers: a 18F-fluorodeoxyglucose positron emission tomography study. CNS Spectr 2010;15:159166.

-39 Henry PK, Murnane KS, Votaw JR, Howell LL: Acute brain metabolic effects of cocaine in rhesus monkeys with a history of cocaine use. Brain Imaging Behav 2010;4:212-219. 